EPJ Web of Conferences 110,01059 (2016)

DOI: $10.1051 /$ epjconf/201611001059

(C) Owned by the authors, published by EDP Sciences, 2016

\title{
ELECTRIC EQUIPMENT DIAGNOSIS BASED ON WAVELET
}

\section{ANALYSIS}

\author{
Sergey A. Stavitsky ${ }^{1}$, Nikolay E. Palukhin ${ }^{1}$, Juri V. Kobenko ${ }^{1, \text { a }}$ and Elena S. Riabova ${ }^{2}$ \\ ${ }^{1}$ National Research Tomsk Polytechnic University, 634050 Tomsk, Russia \\ ${ }^{2}$ Samara State Academy for Humanities and Social Science, 443099 Samara, M. Gorkogo St., 65/67, Russia
}

\begin{abstract}
Due to electric equipment development and complication it is necessary to have a precise and intense diagnosis. Nowadays there are two basic ways of diagnosis: analog signal processing and digital signal processing. The latter is more preferable. The basic ways of digital signal processing (Fourier transform and Fast Fourier transform) include one of the modern methods based on wavelet transform. This research is dedicated to analyzing characteristic features and advantages of wavelet transform. This article shows the ways of using wavelet analysis and the process of test signal converting. In order to carry out this analysis, computer software Mathcad was used and 2D wavelet spectrum for a complex function was created.
\end{abstract}

\section{Introduction}

Power facilities that are used in power engineering feature a complicated electric system with a large number of interrelated elements. The identification of some flaws at the initial stage of their growth in certain elements of this system can be difficult because of indistinct and ambiguously rendered causeeffect relationship between the flaw and measured parameters.

In order to maintain good working condition of electric equipment a series of actions should be taken for engineering service and repair. Repairs of the power-generating equipment can be made with a use of the following repairing strategies: regulation (according to a special plan); mixed (frequency should be in accordance with a plan, volume of works is varied); condition based maintenance; as required. [1]

As a matter of current experience of electric equipment maintenance it is possible to state that each of the mentioned above strategies can be used for various types of equipment. However, nowadays with the use of powerful computer engineering the most promising one is the condition based maintenance strategy. It includes a constant control of equipment performance. And according to the data received from parameter checkout it is possible not only to define the damage but also to set such a working mode that will enable the equipment to function till halting. Thus, this strategy features substantial technical and material advantages.

\section{${ }^{a}$ Corresponding author: serpentis@list.ru}


This strategy is a great deal more complicated and needs experienced personnel and special diagnostic equipment. However, current prices of computer engineering and measurement technology allow installing state-of-the-art diagnostic equipment at minimum expense.

The objective of this paper is to find out the ways of using wavelet analysis and the process of test signal converting.

\section{Problem statement}

The diagnosis of electric equipment is a system of methods and means focused on evaluation of the device's technical state.

The diagnosis consists in a constant readout of necessary signals from transducers and their further handling. [2]

There are two ways of signal handling - analog and digital. However, nowadays due to the level of technology there is no use in analog way of signal handling as the digital one has a number of advantages:

- short time and capability of modern computers to process loads of information in a matter of seconds;

- accuracy of results;

- absence of human factor.

That is why nowadays digital way of signal handling is used. Digital signals are produced from analog ones with the help of sampling operation in a special device - AD converter (analog-to-digital converter). It means that it is reached due to data testing at certain dependent intervals. As a result, the signal is converted into a set of numbers.

Nowadays two main ways of digital signal handling are used: Fourier transform and FFT (Fast Fourier transform). The domain one is FFT.

This method tends to be universally received due to its main advantages: simplicity and accuracy of periodic signal processing. But it also has got a number of disadvantages. The main one is connected with the fact that FFT, as well as Fourier transform, can not define nonperiodic perturbation.

One of the new approaches in digital diagnosis is wavelet analysis.

Wavelet transform as well as Fourier transform is a part of frequency-domain analysis, but in a more general sense. The term "wavelet" means a "short wave". Wavelets look like a short wave function with an amplitude that begins at zero. These functions are traced along parameter axis and are adjusted to expansion and compression operations.

An outstanding feature of wavelet transform that determines its application domain is the capability to process temporary or inhomogeneous signals. In this case the result of the analysis apart from frequency characteristics will contain local coordinates of frequency-domain changes.

Another advantage of a wavelet in comparison to Fourier transform is its ability to transform local signal characteristics including ordinary variable to a high precision.

As a result of wavelet transform of regular signals we get two-dimensional pattern with two predictors: frequency and coordinate that is used for showing the processing result. Thus, the result can be easily seen and it helps to perceive and understand it better.

This research is dedicated to studying the ways of wavelet analysis usage for digital signal processing.

\section{Results and discussion}

The process of signal transformation consists in multiplication of an original signal with a wavelet function. [3] This transformation starts with the scale $a=1$ and goes on with increasing parameters ' $a$ ', i.e. the analysis starts with high frequencies and moves towards low frequencies. The first parameter ' $a$ ' corresponds with the most compressed wavelet. At increasing parameter ' $a$ ' wavelet becomes wider. Wavelet is placed at the beginning of the signal $(\mathrm{t}=0)$, multiplies with the signal, integrates at 
the interval of its starting point and comes to the normal stage at $1 / \sqrt{\mathrm{a}}$. The result of calculation $\mathrm{C}(\mathrm{a}, \mathrm{b})$ is put at the point $(\mathrm{a}=1, \mathrm{~b}=0)$ of time-scale spectrum of transformation. The shift ' $\mathrm{b}$ ' can be observed as time at the moment $\mathrm{t}=0$, and the axis $\mathrm{b}$ repeats the time base of the signal. For a complete implementation of all the input signals, it is important to set the initial and final criteria (certain parameters of the input signal at $\mathrm{t}<0$ и $\mathrm{t}>\mathrm{t}_{\max }$ at wavelet window halfwidth). At single-sided setting of wavelets, the result applies, as a rule, to a temporary position of wavelet window midpoint.

Then the wavelet scaled $a=1$ moves to the right in the position $b$ and the process repeats. Finally, we get the parameter that stands for $\mathrm{t}=\mathrm{b}$ in the line $\mathrm{a}=1$ in time-frequency scale. The process continues until the wavelet reaches the end of the signal. Thus, we get a line of points at time-scale plan for $a=1$.

For calculating the next scale line parameter $a$ is increased to another position at continuous wavelet transform in analytic form $\Delta \mathrm{b} \rightarrow 0$ и $\Delta \mathrm{a} \rightarrow 0$. While making this transform with the help of computer there is an increase of both parameters with a certain interval. Thus, there is a discrete sampling of time-scale plane.

To illustrate this fact, we can take a complicated function including distinct states, in the program package Mathcad (fig. 1)

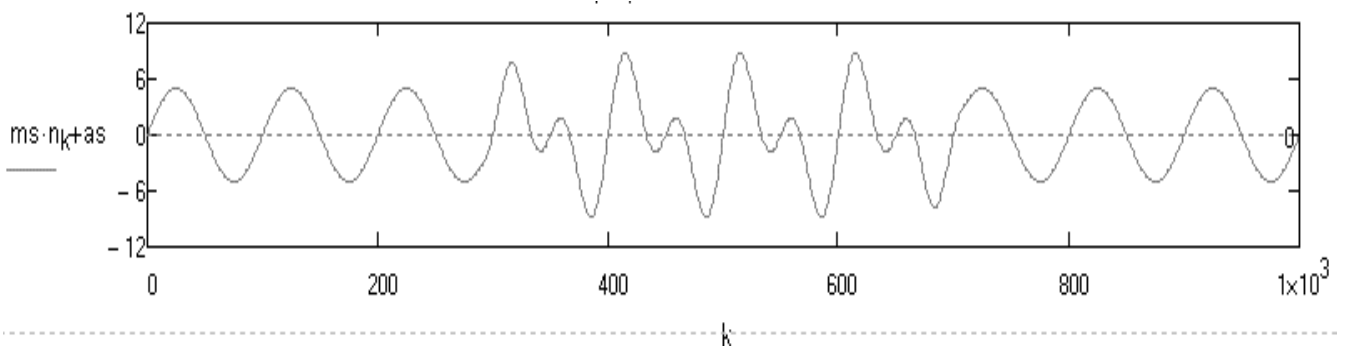

Figure 1. The graphic of the initial function.

For wavelet analysis of input signal we use wavelet Daubechies that is in-line in Mothcad (fig. 2)

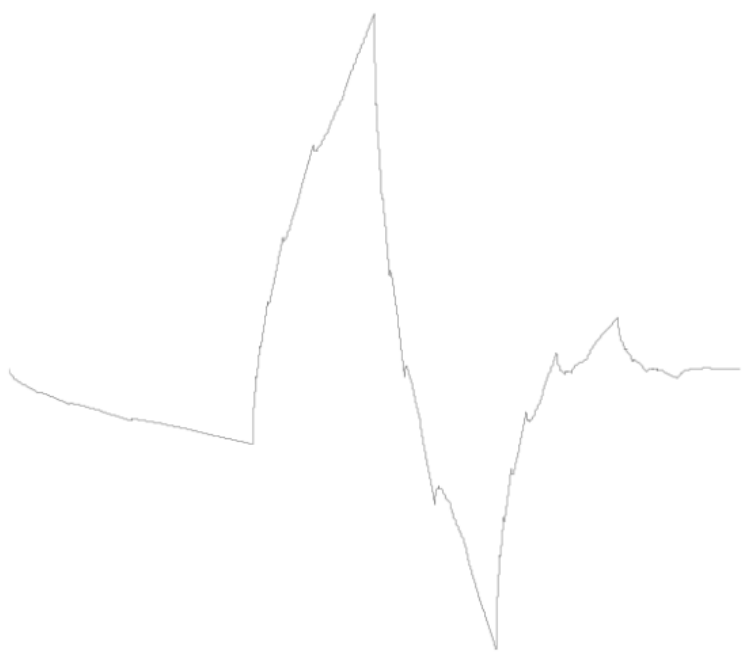

Figure 2. The types of wavelet function. 2D wavelet spectrum (fig. 3). 


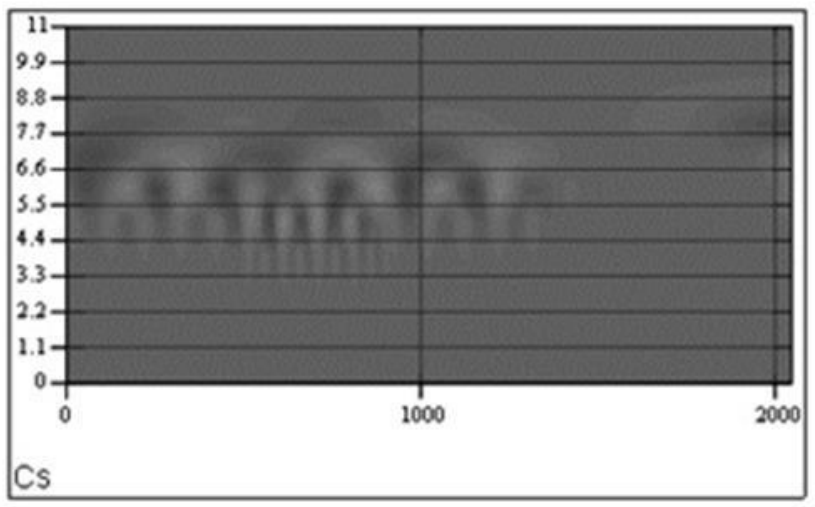

Figure 3. Wavelet spectrum.

\section{Conclusion}

As it is seen from the spectra, the input signal has additional products at two thirds of the signal.

At this part of the section signal product is visualized frequency-doubled. Thus, on the basis of experimental research, wavelet transform is an effective method of non-stationary signal processing.

\section{References}

1. Power-engineering equipment. The system of service and repair of power-engineering equipment [Electronic resource] - URL: http://www.energooborudovanie.ru/, in Russian. Accessed date: 15.04.2014

2. V.P. Kalyavin, L.M. Rybakov, Reliability and diagnostics of electric systems. (Elmore, 2009)

3. Digital signal processing. The theory of signals and systems. Wavelet analysis [Electronic resource] - URL: http:// http://prodav.exponenta.ru/, in Russian. Accessed date: 05.04.2014 